\title{
Anatomical locations of the motor endplates of sartorius muscle for botulinum toxin injections in treatment of muscle spasticity
}

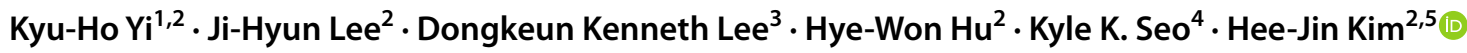

Received: 17 June 2021 / Accepted: 2 August 2021 / Published online: 11 August 2021

(c) The Author(s), under exclusive licence to Springer-Verlag France SAS, part of Springer Nature 2021

\begin{abstract}
Purpose This study aimed to detect the idyllic locations for botulinum neurotoxin injection by analyzing the intramuscular neural distributions of the sartorius muscles.

Methods An altered Sihler's staining was conducted on sartorius muscles (15 specimens). The nerve entry points and intramuscular arborization areas were measured as a percentage of the total distance from the most prominent point of the anterior superior iliac spine (0\%) to the medial femoral epicondyle (100\%).

Results Intramuscular neural distribution were densely detected at $20-40 \%$ and $60-80 \%$ for the sartorius muscles. The result suggests that the treatment of sartorius muscle spasticity requires botulinum neurotoxin injections in particular locations.

Conclusions These locations, corresponding to the locations of maximum arborization, are suggested as the most suggestive points for botulinum neurotoxin injection.
\end{abstract}

Keywords Botulinum neurotoxin $\cdot$ Spasticity $\cdot$ Sartorius muscle $\cdot$ Sihler's staining

\section{Introduction}

The sartorius muscle is the lengthiest muscle in the human anatomy, running over both hip and knee joints. It is a superficially and anteriorly located muscle of the thigh and diagonally runs from the anterior superior iliac spine to the medial side of the proximal tibia at the pes anserinus. The insertion of the sartorius muscle is the superior medial part of the

Kyu-Ho Yi and Ji-Hyun Lee contributed equally to this work.

Hee-Jin Kim

hjk776@yuhs.ac

1 Wonju City Public Health Center, COVID-19 Division, Wonju-Si, Republic of Korea

2 Division in Anatomy and Developmental Biology, Department of Oral Biology, Human Identification Research Institute, BK21 FOUR Project, Yonsei University College of Dentistry, 50-1 Yonsei-ro, Seodaemun-gu, Seoul 03722, Republic of Korea

3 EGDONGAN Skin and Cosmetic Surgery Network, Sinnonhyeon, Seoul, Republic of Korea

4 Modelo Clinic, Seoul, Republic of Korea

5 Department of Materials Science and Engineering, College of Engineering, Yonsei University, Seoul, Republic of Korea tibial bone, close to the medial tibial tubercle. The conjoined tendon of gracilis, semitendinosus, and sartorius muscles is known as the pes anserinus [4].

Spasticity is a main contributor to functional loss in patients with impaired central nervous system, such as in stroke, cerebral palsy, multiple sclerosis, traumatic brain injury, spinal cord injury, and others [2]. Sartorius muscle, as a hip and knee flexor, is one of the commonly involved muscles, and long-lasting spasticity of the muscle results in abnormalities secondary to muscle hyperactivity, affecting lower levels of functions, such as impairment of gait. For ambulatory patients, hip and knee flexor spasticity results in crouched gait, which needs much more strength than in people with normal hip and knee extension. Since hip and knee flexor spasticity occurs in conjunction, surgical and medical treatments are required in sartorius muscle to improve the posture.

Presently, botulinum neurotoxin (BoNT) injection is among the most secure and effective approaches for relieving spasticity $[7,16,24,27,30]$. Since its consequences depend on the amount, the recommended BoNT levels should be sufficient in sartorius muscle at the area of neuromuscular junctions [37]. However, BoNT overdose may cause the neurotoxin to spread to adjacent muscles and cause undesirable paralysis $[11,15,18]$. Thus, to lessen the adverse effects and 
to maximize its clinical efficacy, BoNT must be injected near the neural arborized areas. Numerous studies have revealed the anatomical location of neuromuscular junctional areas of many different muscles $[6,12,18,26,29]$. The consequence of neural arborized area-targeted injection, in which most neuromuscular junctions are located, has been established in clinical studies on biceps brachii and psoas major muscles $[5,8]$. Intramuscular injection in neural arborized areas of biceps brachii and psoas major resulted in a much higher volume reduction than the control $[5,8]$.

Studies that explain BoNT injection locations by navigating intramuscular neural distribution with naked eye dissection have limits, such as perceiving minuscule nerves and the likelihood of neural damage [17, 21, 25, 41-49].

Moreover, earlier studies have pronounced trouble in precisely detecting the tiny nerves $[29,34,40]$. These restrictions can be disregarded when proceeding with Sihler's staining, which is a whole-mount nerve staining that changes the muscle translucent while stained nerves.

Sihler's staining is an efficient method to precisely demonstrate the intramuscular nerve distribution without damaging the nerve itself, and offers a discrete and comprehensive outline of the nerve distribution.

The objective of this study was to determine the intramuscular nerve distribution of sartorius muscle using Sihler's staining and to suggest BoNT injection points for treating hip and knee flexor spasticity.

\section{Methods and materials}

\section{Subjects}

Informed consent and approval were obtained from the families of the cadavers before the dissections were performed. All cadavers used in this study were legally donated and approved from ethics committee of the Surgical Anatomy Education Center, Yonsei University College of Medicine (approval code 20-009; approval date: May 5th, 2020). Fifteen sartorius muscles (eight right sides and seven left sides; eight male and seven female specimens) from 10 cadavers (five women and five men with a mean age of 74.2 years; range, 63-84 years) were dissected to explore nerve entry points, and Sihler's staining was performed to detect intramuscular neural distribution. The sartorius muscles were clean with no clues of an operation history or diseases.

The Sihler's staining procedure requires multiple stages to acquire the image of the intramuscular neural distribution [20,33, 35-39]. After the staining procedures, the sartorius muscles were equally divided into 10 , each represented division of $10 \%$, respectively to the total length. The origin of the sartorius muscles, nerve entry points, and intramuscular neural arborized locations were measured as percentages from the anterior superior iliac spine $(0 \%)$ to the medial femoral epicondyle (100\%) (Fig. 1).

The stages of the Sihler's staining of the sartorius muscle are described in Fig. 2.

\section{Modified Sihler's staining}

Fixation stage: The extracted sartorius muscles underwent fixation for 30 days in a container filled with $10 \%$

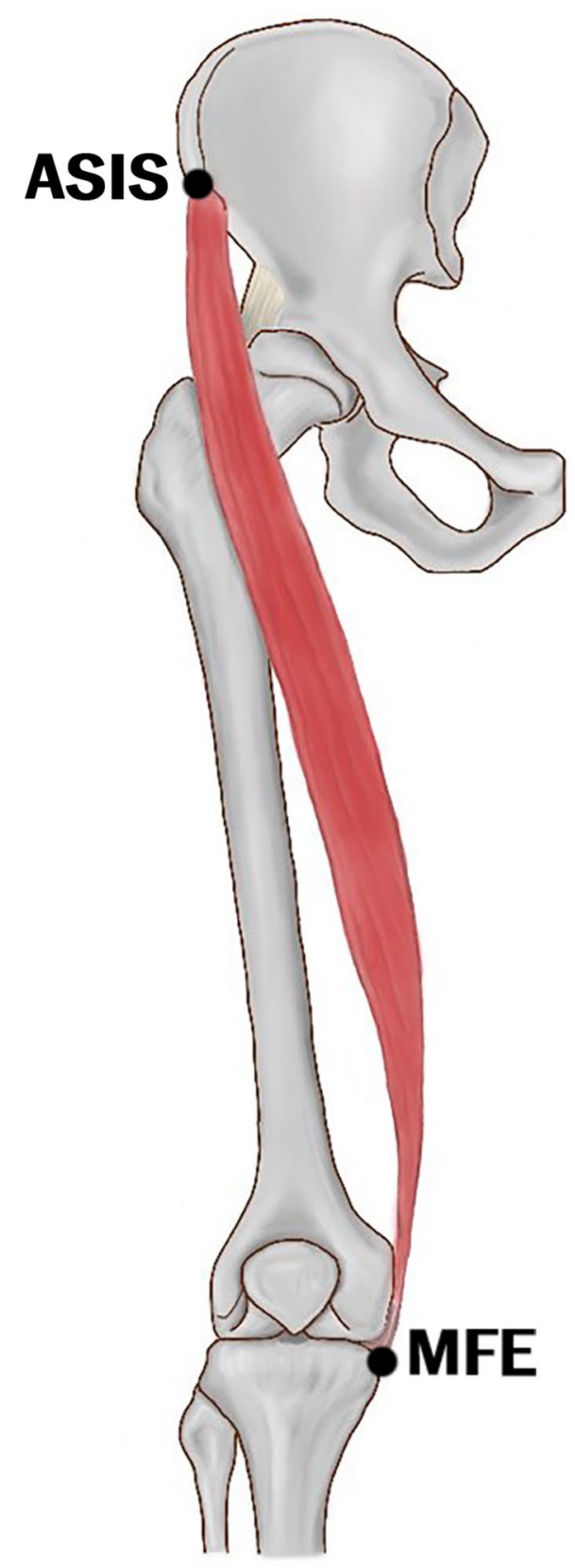

Fig. 1 Sartorius muscles were harvested from the anterior superior iliac spine (ASIS) $(0 \%)$ to the medial femoral epicondyle (MFE) $(100 \%)$ 


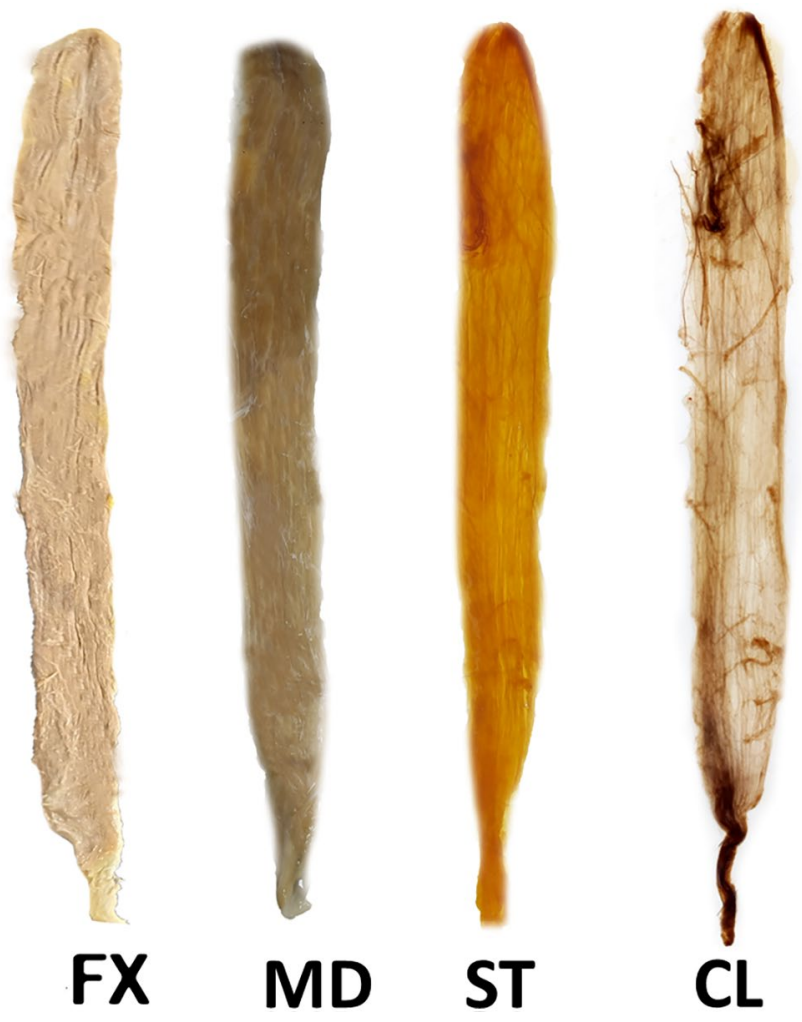

Fig. 2 The sartorius muscle underwent modified Sihler's staining. The method consists of the procedures of fixation (FX), maceration and depigmentation (MD), decalcification, staining (ST), and clearing (CL)

unneutralized formalin. The formalin solution was replaced each time it turned hazy.

Maceration and depigmentation stages: Once fixation was completed, the sartorius muscles were placed in flowing water for $1 \mathrm{~h}$. Next, the sartorius muscles were positioned in a $3 \%$ aqueous potassium hydroxide solution with hydrogen peroxide for 2 weeks.

Decalcification stage: The macerated sartorius muscle was immersed in a container filled with Sihler's solution I, which is composed of glycerin, aqueous chloral hydrate, and glacial acetic acid, for 3 days.

Staining stage: Sufficiently decalcified sartorius muscles were later stained by placing them for a day in Sihler's solution II, composed of glycerin, acetic acid, and aqueous chloral hydrate.

Destaining stage: Stained sartorius muscles were immersed for 3-5 h in Sihler's solution I once again for sartorius muscle tissue destaining. The immersion was terminated before the nerves were destained.

Neutralization stage: Destained sartorius muscle was prepared in flowing water for $30 \mathrm{~min}$. Afterwards the muscles were immersed in $0.05 \%$ lithium carbonate solution for $30 \mathrm{~min}$.
Clearing stage: Neutralized sartorius muscles were placed in containers with increasing level of glycerin concentration within 5 days. Throughout this procedure, the concentration level was progressively increased in $20 \%$ increments to $40-100 \%$.

\section{Results}

\section{Location of nerve entry points}

In all cases, the sartorius muscle was innervated by the femoral nerve. The numbers of the nerves penetrating the muscle was 1-3, with no significant differences between the gender and sides. Four cases had three nerve entry points, nine cases had two nerve entry points, and two cases had one nerve entry point. The distance of all nerve entry points was located at $10-30 \%$ from the anterior superior iliac spine $(0 \%)$ to the medial femoral epicondyle (100\%).

\section{Intramuscular arborization patterns}

Eleven out of 15 sartorius muscles had the most nerve arborizations appear at 2 regions, located at proximal 20-40\% and distal $60-80 \%$. Three had most nerve arborization at proximal $30-40 \%$ and distal $60-80 \%$, and one had it at proximal 30-40\% and distal 60-70\%. All specimens had the most distally located intramuscular nerve ending at around 80-90\% of each muscle (Fig. 3). The intramuscular arborization patterns had no significant differences between the gender and sides.

\section{Discussion}

The sartorius muscle is innervated by the femoral nerve, provided by the nerve roots L2-L4 [4]. The nerve entry point, where the nerve pierces the muscle, was at its proximal end. The most prevalent spasticity patterns in the lower extremities are hip flexion and adduction, knee flexion, equinovarus foot, and big toe hyperextension that impedes the gait of affected patients [14]. Since the sartorius is the only hip and knee flexor muscle, it is commonly targeted for BoNT injections to ease spasticity. BoNT injection in the sartorius muscle is not only for spasticity treatment, as many studies have performed BoNT injection to effectively prevent muscle contractures in total hip and knee arthroplasties and other surgeries $[3,28]$. Awaad et al. [1] reported the functional contribution of BoNT injection into the sartorius muscle in the treatment of postoperative stiff hips. Hamdy et al. [9, 10] used BoNT in femoral lengthening surgery and concluded that it improved the post-operative pain reduction. On the 


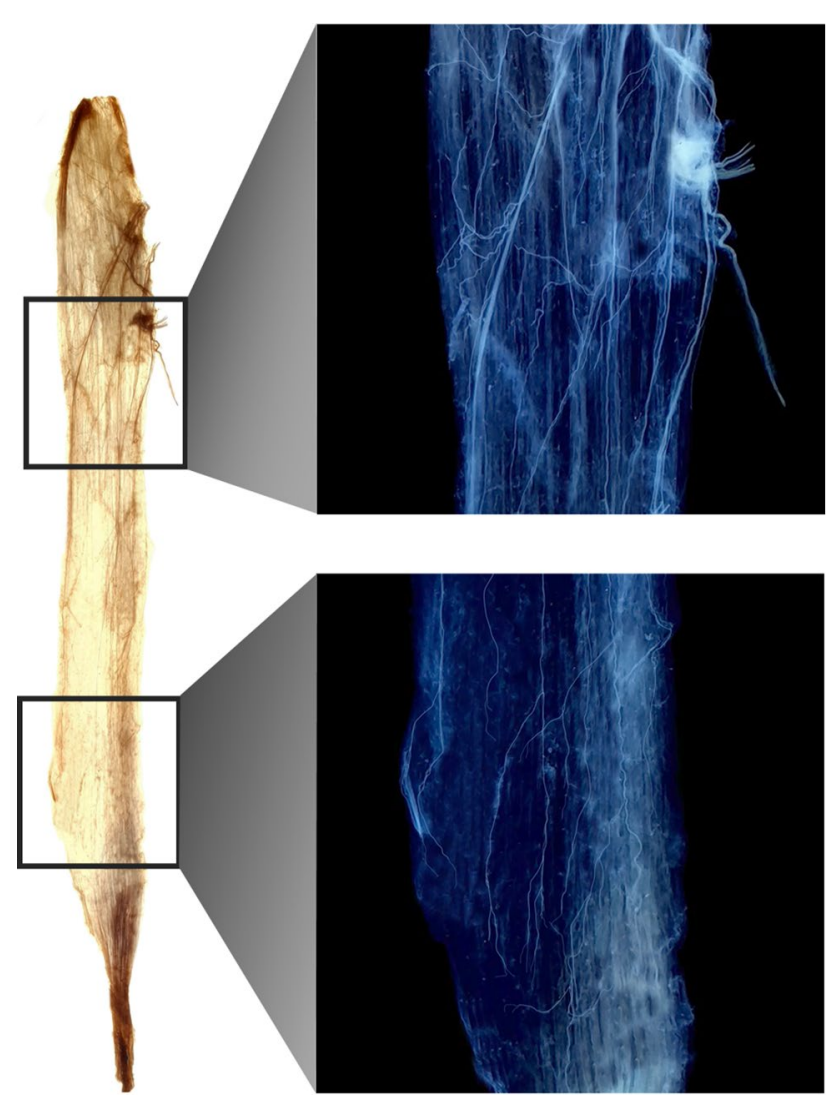

Fig. 3 A Sihler's-stained sartorius muscle with enlarged images of intramuscular neural distributions. Intramuscular nerve pattern and entry points were revealed by dissection and staining

contrary, Park et al. [23] reported that BoNT had no significant impact on pain reduction after lengthening osteotomy. However, their study had the limitation of using smaller doses than Hamdy et al. and had targeted only the proximal part of the sartorius muscles with no clear injection points.

The major therapeutic effects of BoNT are by impeding muscle contractions at the neuromuscular junction and cutting off the vicious cycle of pain [22, 31, 32]. However, there is still a risk of damaging the nerve trunks when BoNT was not injected into the neural arborized area, even if it is minimally invasive compared to surgical procedures. Likewise, substantial BoNT injections have resulted in the formation of antibodies which lowers its effectiveness [11, 15, 18]. Consequently, to increase the effectiveness and reduce the negative effects, a smaller amount of BoNT needs to be injected directly into the neural arborized areas, where most neuromuscular junctions are assumed to be located. Its efficacy was recognized in a clinical study on psoas major and biceps brachii muscles. Intramuscular neural arborized area-targeted injection in these studies resulted in a higher reduction in muscle volume than conventional injection methods $[5,8]$.

Ultrasound and electromyography devices are used to detect the sartorius muscle $[13,19]$. Ultrasound imaging detection with electromyography guidance has been demonstrated to be an ideal method for avoiding side effects associated with BoNT injections. Overdose of BoNT may cause the neurotoxin to spread to adjacent muscles causing undesirable paralysis and lead to the development of antibodies against the toxin. Consequently, attempting to prevent toxin resistance from antibody production by exactly locating sartorius muscle with smaller doses is an important consideration $[11,15,18]$.

Currently, there is no standardized injection point for BoNT treatment of the sartorius muscle. The amount of BoNT should be adequate to introduce a sufficient toxin level in the arborized area of neural distribution. This study used the Sihler's staining method, which provides a possible solution to resolve the limitations of manual dissection. The application of Sihler's staining to sartorius muscle will facilitate accurate and thorough understanding of the neural distribution. This study performed Sihler's staining to reveal the intramuscular neural distribution of the sartorius muscle to determine the most suggestive BoNT injection point (Fig. 4). 


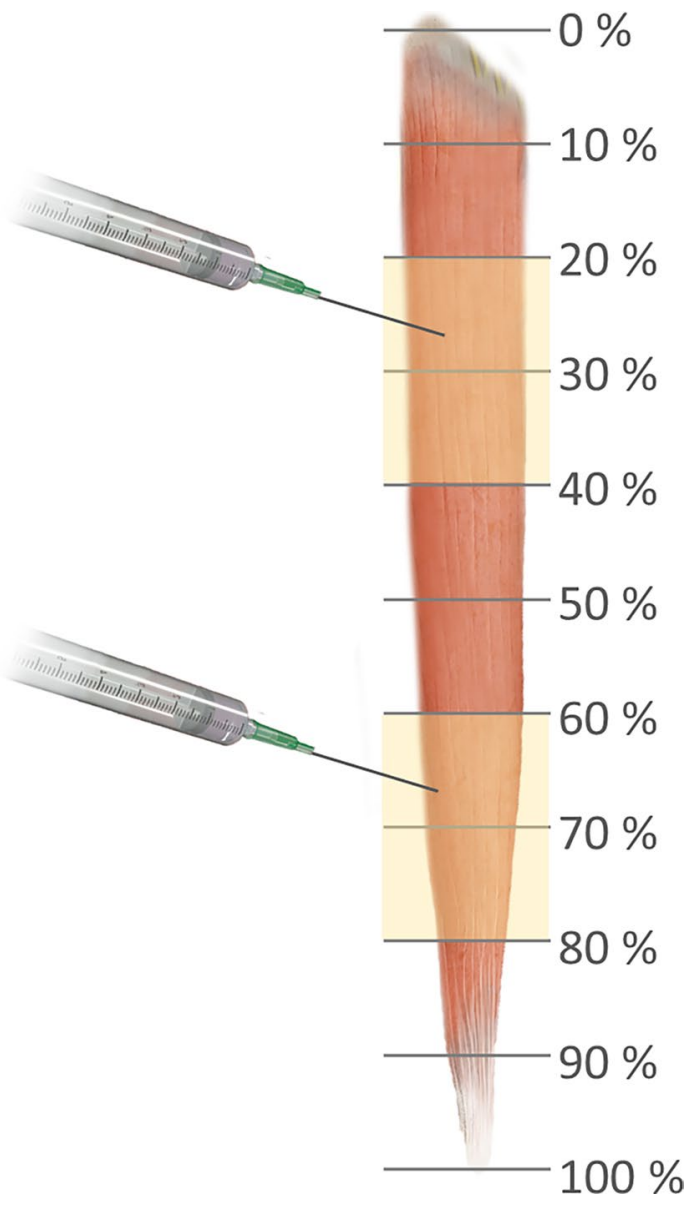

Fig. 4 Recommendation for electromyography or BoNT injection of the sartorius muscle. The ultimate suggestions were achieved in relatively to the anterior superior iliac spine to the medial femoral epicondyle. The sartorius muscles demonstrated arborization neural distribution at $20-40 \%$ and $60-80 \%$ (yellow boxes)

Acknowledgements The authors sincerely thank those who donated their bodies to science so that anatomical research could be performed. Results from such research can potentially increase mankind's overall knowledge that can then improve patient care. Therefore, these donors and their families deserve our highest gratitude. The authors thank Eun-Byul Yi from Eonbuk elementary school for illustrations. This work was supported by the National Research Foundation of Korea (NRF) grant funded by the Korean government (MSIP) (NRF-2019R1C1C1010776).

Funding None of the authors claim financial support from commercial, academic, or political organizations or people regarding this study.

\section{Declarations}

Conflict of interest I acknowledge that I have considered the conflict of interest statement included in the "Author Guidelines." I hereby certify that, to the best of my knowledge, that no aspect of my current personal or professional situation might reasonably be expected to significantly affect my views on the subject I am presenting.

\section{References}

1. Awaad Y, Rizk T, Abak AA, Kabiruddin K, Khoshhal KI (2013) Botulinum toxin-A in postoperative pediatric stiff hips. J Taibah Univ Med Sci 8(3):187-191

2. Barnes MP, Kent RM, Semlyen JK, McMullen KM (2003) Spasticity in multiple sclerosis. Neurorehabil Neural Repair 17(1):66-70

3. Bhave A, Zywiel MG, Ulrich SD, McGrath MS, Seyler TM, Marker DR, Delanois RE, Mont MA (2009) Botulinum toxin type A injections for the management of muscle tightness following total hip arthroplasty: a case series. J Orthop Surg Res 4:34

4. Buckland A, Pan WR, Dhar S, Edwards G, Rozen WM, Ashton MW, Taylor GI (2009) Neurovascular anatomy of sartorius muscle flaps: implications for local transposition and facial reanimation. Plast Reconstr Surg 123(1):44-54

5. Van Campenhout A, Verhaegen A, Pans S, Molenaers G (2013) Botulinum toxin type A injections in the psoas muscle of children with cerebral palsy: muscle atrophy after motor end plate-targeted injections. Res Dev Disabil 34(3):1052-1058

6. Chantelot C, Feugas C, Guillem P, Chapnikoff D, Remy F, Fontaine $C$ (1999) Innervation of the medial epicondylar muscles: an anatomic study in 50 cases. Surg Radiol Anat 21(3):165-168

7. Diserens K, Ruegg D, Kleiser R, Hyde S, Perret N, Vuadens P, Fornari E, Vingerhoets F, Seitz RJ (2010) Effect of repetitive arm cycling following botulinum toxin injection for poststroke spasticity: evidence from FMRI. Neurorehabil Neural Repair 24(8):753-762

8. Gracies JM, Lugassy M, Weisz DJ, Vecchio M, Flanagan S, Simpson DM (2009) Botulinum toxin dilution and endplate targeting in spasticity: a double-blind controlled study. Arch Phys Med Rehabil 90(1):9-16 (e12)

9. Hamdy RC, Montpetit K, Aiona MD, MacKenzie WG, van Bosse HJ, Narayanan U, Raney EM, Chafetz RS, Thomas SE, Weir S, Gregory S, Yorgova P, Takahashi S, Rinaldi M, Zhang X, DahanOliel N (2016) Safety and efficacy of botulinum toxin A in children undergoing lower limb lengthening and deformity correction: results of a double-blind, multicenter, Randomized Controlled Trial. J Pediatr Orthop 36(1):48-55

10. Hamdy RC, Montpetit K, Raney EM, Aiona MD, Fillman RR, MacKenzie W, McCarthy J, Chafetz RS, Thomas SS, Tamayo CM, Littleton AG, Ruck-Gibis J, Takahashi SN, Rinaldi M, Finley GA, Platt RW, Dahan-Oliel N (2009) Botulinum toxin type A injection in alleviating postoperative pain and improving quality of life in lower extremity limb lengthening and deformity correction: a pilot study. J Pediatr Orthop 29(5):427-434

11. Hsu TS, Dover JS, Arndt KA (2004) Effect of volume and concentration on the diffusion of botulinum exotoxin A. Arch Dermatol 140(11):1351-1354

12. Hwang K, Jin S, Hwang SH, Lee KM, Han SH (2007) Location of nerve entry points of flexor digitorum profundus. Surg Radiol Anat 29(8):617-621

13. Johnson CE, Basmajian JV, Dasher W (1972) Electromyography of sartorius muscle. Anat Rec 173(2):127-130

14. Johnson CA, Burridge JH, Strike PW, Wood DE, Swain ID (2004) The effect of combined use of botulinum toxin type A and functional electric stimulation in the treatment of spastic drop foot after stroke: a preliminary investigation. Arch Phys Med Rehabil 85(6):902-909

15. Kinnett D (2004) Botulinum toxin A injections in children: technique and dosing issues. Am J Phys Med Rehabil 83(10 Suppl):S59-64

16. Lam TI, Bingham D, Chang TJ, Lee CC, Shi J, Wang D, Massa S, Swanson RA, Liu J (2013) Beneficial effects of minocycline and botulinum toxin-induced constraint physical therapy following 
experimental traumatic brain injury. Neurorehabil Neural Repair 27(9):889-899

17. Lee JH, Lee BN, An X, Chung RH, Han SH (2011) Location of the motor entry point and intramuscular motor point of the tibialis posterior muscle: for effective motor point block. Clin Anat 24(1):91-96

18. Lepage D, Parratte B, Tatu L, Vuiller F, Monnier G (2005) Extra- and intramuscular nerve supply of the muscles of the anterior antebrachial compartment: applications for selective neurotomy and for botulinum toxin injection. Surg Radiol Anat 27(5):420-430

19. Malherbe K (2019) A rare ultrasound case report: intramuscular tear of the sartorius muscle. Ultrasound J 11(1):17

20. Mu L, Sanders I (2010) Sihler's whole mount nerve staining technique: a review. Biotech Histochem 85(1):19-42

21. Oddy MJ, Brown C, Mistry R, Eastwood DM (2006) Botulinum toxin injection site localization for the tibialis posterior muscle. $\mathrm{J}$ Pediatr Orthop B 15(6):414-417

22. Ondo WG, Vuong KD, Derman HS (2004) Botulinum toxin A for chronic daily headache: a randomized, placebo-controlled, parallel design study. Cephalalgia 24(1):60-65

23. Park H, Shin S, Shin HS, Kim HW, Kim DW, Lee DH (2016) Is botulinum toxin type A a valuable adjunct during femoral lengthening? A randomized trial. Clin Orthop Relat Res 474(12):2705-2711

24. Rameckers EA, Speth LA, Duysens J, Vles JS, Smits-Engelsman BC (2009) Botulinum toxin-A in children with congenital spastic hemiplegia does not improve upper extremity motor-related function over rehabilitation alone: a randomized controlled trial. Neurorehabil Neural Repair 23(3):218-225

25. Rha DW, Yi KH, Park ES, Park C, Kim HJ (2016) Intramuscular nerve distribution of the hamstring muscles: application to treating spasticity. Clin Anat 29(6):746-751

26. Roberts C, Crystal R, Eastwood DM (2006) Optimal injection points for the neuromuscular blockade of forearm flexor muscles: a cadaveric study. J Pediatr Orthop B 15(5):351-355

27. Rosales RL, Kong KH, Goh KJ, Kumthornthip W, Mok VC, Delgado-De Los Santos MM, Chua KS, Abdullah SJ, Zakine B, Maisonobe P, Magis A, Wong KS (2012) Botulinum toxin injection for hypertonicity of the upper extremity within 12 weeks after stroke: a randomized controlled trial. Neurorehabil Neural Repair 26(7):812-821

28. Seyler TM, Jinnah RH, Koman LA, Marker DR, Mont MA, Ulrich SD, Bhave A (2008) Botulinum toxin type A injections for the management of flexion contractures following total knee arthroplasty. J Surg Orthop Adv 17(4):231-238

29. Sheverdin VA, Hur MS, Won SY, Song WC, Hu KS, Koh KS, Kim HJ (2009) Extra- and intra-muscular nerves distributions of the triceps surae muscle as a basis for muscle resection and botulinum toxin injections. Surg Radiol Anat 31(8):615-621

30. Sun SF, Hsu CW, Sun HP, Hwang CW, Yang CL, Wang JL (2010) Combined botulinum toxin type A with modified constraintinduced movement therapy for chronic stroke patients with upper extremity spasticity: a randomized controlled study. Neurorehabil Neural Repair 24(1):34-41

31. Tepper SJ (2004) Treatment of headache pain with botulinum neurotoxins. Pain Pract 4(Suppl 1):S38-46

32. Troost BT (2004) Botulinum toxin type A (Botox) in the treatment of migraine and other headaches. Expert Rev Neurother 4(1):27-31

33. Won SY, Cho YH, Choi YJ, Favero V, Woo HS, Chang KY, Hu KS, Kim HJ (2015) Intramuscular innervation patterns of the brachialis muscle. Clin Anat 28(1):123-127
34. Won SY, Choi DY, Lee JG, Yoon KH, Kwak HH, Hu KS, Kim HJ (2010) Intramuscular communicating branches in the flexor digitorum profundus: dissection and Sihler's staining. Surg Radiol Anat 32(3):285-289

35. Won SY, Hur MS, Rha DW, Park HD, Hu KS, Fontaine C, Kim HJ (2010) Extra- and intra-muscular nerve distribution patterns of the muscles of the ventral compartment of the forearm. Am J Phys Med Rehabil 89(8):644-652

36. Won SY, Kim DH, Yang HM, Park JT, Kwak HH, Hu KS, Kim HJ (2011) Clinical and anatomical approach using Sihler's staining technique (whole mount nerve stain). Anat Cell Biol 44(1):1-7

37. Won SY, Rha DW, Kim HS, Jung SH, Park ES, Hu KS, Kim HJ (2012) Intramuscular nerve distribution pattern of the adductor longus and gracilis muscles demonstrated with Sihler staining: guidance for botulinum toxin injection. Muscle Nerve 46(1):80-85

38. Yang HM, Won SY, Kim HJ, Hu KS (2013) Sihler staining study of anastomosis between the facial and trigeminal nerves in the ocular area and its clinical implications. Muscle Nerve 48(4):545-550

39. Yang HM, Won SY, Lee YI, Kim HJ, Hu KS (2014) The Sihler staining study of the infraorbital nerve and its clinical complication. J Craniofac Surg 25(6):2209-2213

40. Yang HM, Woo YJ, Won SY, Kim DH, Hu KS, Kim HJ (2009) Course and distribution of the lingual nerve in the ventral tongue region: anatomical considerations for frenectomy. J Craniofac Surg 20(5):1359-1363

41. Yi KH, Choi YJ, Cong L, Lee KL, Hu KS, Kim HJ (2020) Effective botulinum toxin injection guide for treatment of cervical dystonia. Clin Anat 33(2):192-198

42. Yi KH, Cong L, Bae JH, Park ES, Rha DW, Kim HJ (2017) Neuromuscular structure of the tibialis anterior muscle for functional electrical stimulation. Surg Radiol Anat 39(1):77-83

43. Yi KH, Kim HJ (2020) Is variation in posterior tibial veins a risk factor for deep-vein thrombosis? Clin Anat. https://doi.org/10. 1002/ca.23691

44. Yi KH, Lee HJ, Choi YJ, Lee JH, Hu KS, Kim HJ (2020) Intramuscular neural distribution of rhomboid muscles: evaluation for botulinum toxin injection using modified Sihler's method. Toxins (Basel) 12(5):289

45. Yi KH, Lee HJ, Choi YJ, Lee K, Lee JH, Kim HJ (2020) Anatomical guide for botulinum neurotoxin injection: application to cosmetic shoulder contouring, pain syndromes, and cervical dystonia. Clin Anat. https://doi.org/10.1002/ca.23690

46. Yi KH, Lee KL, Lee JH, Hu HW, Lee K, Seo KK, Kim HJ (2020) Guidelines for botulinum neurotoxin injections in piriformis syndrome. Clin Anat. https://doi.org/10.1002/ca.23711

47. Yi KH, Lee HJ, Lee JH, Lee KL, Kim HJ (2021) Effective botulinum neurotoxin injection in treating iliopsoas spasticity. Clin Anat 34(3):431-436

48. Yi KH, Lee HJ, Lee JH, Seo KK, Kim HJ (2021) Application of botulinum neurotoxin injections in TRAM flap for breast reconstruction: intramuscular neural arborization of the rectus abdominis muscle. Toxins (Basel) 13(4):269

49. Yi KH, Rha DW, Lee SC, Cong L, Lee HJ, Lee YW, Kim HJ, Hu KS (2016) Intramuscular nerve distribution pattern of ankle invertor muscles in human cadaver using Sihler stain. Muscle Nerve 53(5):742-747

Publisher's Note Springer Nature remains neutral with regard to jurisdictional claims in published maps and institutional affiliations. 